\title{
Reliable identification at the species level of Brucella isolates with MALDI-TOF-MS
}

\author{
Florigio Lista ${ }^{3}$, Frans AG Reubsaet ${ }^{2}$, Riccardo De Santis ${ }^{3}$, Rene R Parchen ${ }^{1}$, Ad L de Jong ${ }^{1}$, Jasper Kieboom', \\ Anton $L$ van der Laaken ${ }^{1}$, Ingrid Al Voskamp-Visser ${ }^{1}$, Silvia Fillo ${ }^{3}$, Hugo-Jan Jansen ${ }^{4}$, Jan Van der Plas ${ }^{4}$ and \\ Armand Paauw ${ }^{1 *}$
}

\begin{abstract}
Background: The genus Brucella contains highly infectious species that are classified as biological threat agents. The timely detection and identification of the microorganism involved is essential for an effective response not only to biological warfare attacks but also to natural outbreaks. Matrix-assisted laser desorption/ionization time-offlight mass spectrometry (MALDI-TOF-MS) is a rapid method for the analysis of biological samples. The advantages of this method, compared to conventional techniques, are rapidity, cost-effectiveness, accuracy and suitability for the high-throughput identification of bacteria. Discrepancies between taxonomy and genetic relatedness on the species and biovar level complicate the development of detection and identification assays.
\end{abstract}

Results: In this study, the accurate identification of Brucella species using MALDI-TOF-MS was achieved by constructing a Brucella reference library based on multilocus variable-number tandem repeat analysis (MLVA) data. By comparing MS-spectra from Brucella species against a custom-made MALDI-TOF-MS reference library, MALDITOF-MS could be used as a rapid identification method for Brucella species. In this way, $99.3 \%$ of the 152 isolates tested were identified at the species level, and B. suis biovar 1 and 2 were identified at the level of their biovar. This result demonstrates that for Brucella, even minimal genomic differences between these serovars translate to specific proteomic differences.

Conclusions: MALDI-TOF-MS can be developed into a fast and reliable identification method for genetically highly related species when potential taxonomic and genetic inconsistencies are taken into consideration during the generation of the reference library.

\section{Background}

The genus Brucella contains highly infectious species that have been found to cause infections in a wide variety of mammals. Most Brucella species have a narrow host range. Infection in humans arises from direct or indirect contact with infected animals or through consumption of contaminated meat or dairy products [1]. Diagnostic laboratory workers are also at risk; $2 \%$ of all cases of brucellosis are laboratory acquired. Person-to-person transmission is extremely rare [1-3]. Characteristically, Brucella species have a low infectious dose and are capable of transmission via aerosols, and the treatment of infections is lengthy with a risk of complications. For

\footnotetext{
* Correspondence: armand.paauw@tno.nl

'Department of Earth, Environmental, and Life Sciences, TNO, Lange Kleiweg

137, Rijswijk, P.O Box 45, The Netherlands

Full list of author information is available at the end of the article
}

these reasons, Brucella is classified as a potential warfare threat agent, and Brucella suis has been weaponized in the past by the United States, the former Soviet Union, and China [4].

Brucella species belong to the family Brucellaceae in the order Rhizobiales of the class Alphaproteobacteria and are small, non-motile Gram-negative rods. Until recently, six species, some of which may be subdivided into biovars, were assigned to the Brucella genus. These species are Brucella abortus (seven biovars), Brucella melitensis (three biovars), Brucella suis (five biovars), Brucella ovis, Brucella canis, and Brucella neotomae. However, four new species have recently been described. Three of these species were isolated from sea mammals and 'wild' mammals: Brucella ceti, Brucella pinnipedialis, and Brucella microti [5-10]. Finally, a new species, Brucella inopinata, was isolated from a breast implant 
(strain BO1) and from a lung biopsy (strain BO2) $[11,12]$.

The Brucella species primarily considered to be pathogenic for humans are B. melitensis, B. suis (biovars 1, 3, and 4), B. abortus, and sporadically B. canis $[1,2,13]$. $B$. suis biovars 2 and 5 are considered not to be human pathogens because no human cases have been documented for these agents [13].

The DNA-DNA hybridization results suggest that the classification system used for Brucella is open to debate. Among the different Brucella species, the DNA-DNA hybridization relatedness varies from $87 \%$ to $99 \%$, indicating that the Brucella species may actually be considered a single species [13-15]. However, the traditional nomenclature was maintained because the specific host range and pathogenicity differ among the Brucella species [1]. The conventional methods used to identify Brucella isolates are complex, labor-intensive, and time consuming. In addition, Brucella is a potential health hazard to laboratory personnel. Traditionally, the identification of Brucella species is mainly based on host specificity, pathogenicity, and minor phenotypic differences that are determined using several separate tests, which include tests for the oxidation of carbohydrate and amino acid substrates, phage sensitivity, $\mathrm{CO}_{2}$ requirement, $\mathrm{H}_{2} \mathrm{~S}$ production, serum agglutination, and growth in the presence of thionine and basic fuchsine [1]. The scheme to discriminate to the level of biovars is inconclusive because the biological differences between the biovars described are limited, and the interpretation of the results can be subjective [13]. In addition, some Brucella isolates appear unable to be typed [13].

DNA-based approaches have been widely introduced to identify microorganisms, including Brucella species. A relatively rapid approach is the 'Bruce-ladder', a multiplex PCR that is able to distinguish the six classical species $[13,16]$. To complement the 'Bruce-ladder', a single PCR was added to distinguish the marine mammal-derived Brucellae as well. This method, called $b p 26$ PCR, is based on the IS711 $[13,16]$.

Another method, mainly developed for the epidemiological investigation of outbreaks, is multilocus variablenumber tandem repeat analysis (MLVA). MLVA is based on the differences in the number of tandem repeats in several loci of the bacterial chromosome [17]. The MLVA developed for Brucella has been proven to be a reliable, reproducible, and highly discriminatory method that is able to classify all of the Brucella strains $[13,18-20]$. In this study, we the previously described MLVA-16 assay to identify Brucella species was used [13,18-20].

Genomic studies have shown that the nomenclature for several Brucella species is not consistent if the genetic relationships among species are considered to be the gold standard for discriminating between species [20]. For example, $B$. cet $i$ is divided into two separate groups, one of which is more closely related to $B$. pinnipedialis than to the other group of B. ceti [20]. Additionally, B. suis biovar 5 is more related to $B$. ceti, $B$. neotomae, B. pinnipedialis and $B$. ovis than to the other B. suis biovars [20].

The timely detection and rapid identification of the microorganisms involved are essential for the mosteffective response to an infectious disease outbreak, regardless of whether the outbreak is natural or deliberate. This rapid identification is necessary not only to treat patients effectively but also to establish outbreak management, source tracing, and threat analyses.

Matrix-assisted laser desorption/ionization time-offlight mass spectrometry (MALDI-TOF-MS) is a rapid method used to analyze biological differences in microorganisms. MALDI-TOF-MS emerged as a new diagnostic tool in established microbiological laboratories [21]. The advantages of MALDI-TOF-MS over conventional techniques are that it is a fast, cost-effective, accurate method, which is suitable for the high-throughput identification of bacteria by less-skilled laboratory personnel because preliminary identification steps are unnecessary [21-24]. The bacteria are identified by comparing the obtained MS spectra to the MS spectra or profiles of MS spectra from a reference library. Hence, the reliability of the identification is based on the content and quality of this library, among other factors. Recently, a reference library to identify Brucella species was constructed using 12 Brucella strains, but using this 'Brucella library', the discrimination was insufficient for identification at the species level [25]. In contrast, reliable identification at the species level was shown for other genetically closely related species, such as Fransicella species, Bacillus species, and species from the Burkholderia cepacia complex [26-28].

The aim of this study was to improve identification using MALDI-TOF-MS at the species level of Brucella. Therefore, a custom reference library was constructed with strains that represent the known genetic variation of Brucella at the species and biovar level according to MLVA. Subsequently, this custom reference library was evaluated using 152 Brucella isolates that were identified using MLVA.

\section{Methods}

\section{Bacterial strains}

Seventeen of the 170 isolates included in this study are reference strains representing the classical Brucella species, and only the classical reference strain for B. suis biovar 4 is missing (Additional file 1: Table S1) [1]. The 170 isolates included in the study were all typed using MLVA [19]. The Brucella isolates originated from K. Walravens, Veterinary and Agrochemical Research 
Centre (CODA/CERVA), Operational Direction of Bacterial Diseases, Unit of Pathology, Brussels, Belgium, and from the department of Bacteriology of the National Institute for Public Health and the Environment (RIVM), Bilthoven, The Netherlands (Brucella isolated from humans and reference strains). The human isolates from the RIVM were all, except two, isolated from patients in The Netherlands between 1969 and 2008. The strains, together with additional information, are shown in Additional file 1: Table S1.

\section{MLVA analysis}

The target DNA for polymerase chain reaction (PCR) assays was extracted by heating bacterial suspensions in sterilized, demineralized water for $90 \mathrm{~min}$ at $95^{\circ} \mathrm{C}$. The amplification of the different variable-number tandem repeats (VNTR) was performed as previously described [18,19,29-31]. Moreover, as described by Al Dahouk et al., an additional VNTR was added to the initial MLVA-15 [18,19,29,30].

The PCR amplification was performed in 15- $\mu$ l volumes containing $1 \mathrm{U}$ FastStart Taq polymerase (Roche), $1 \times$ PCR Roche reaction buffer (10 mM Tris$\mathrm{HCl}, 2.5 \mathrm{mM} \mathrm{MgCl}_{2}$, and $50 \mathrm{mM} \mathrm{KCl}$ at $\mathrm{pH} 8.3$ ), $0.2 \mathrm{mM}$ dNTPs (Roche) and $0.3 \mu \mathrm{M}$ of each flanking primer. Thermal cycling, conducted on a Peltier Thermal Cycler DNA Engine DYAD (MJ Research), was performed as follows: an initial heating at $95^{\circ} \mathrm{C}$ for $5 \mathrm{~min}$ followed by 35 cycles of denaturation at $95^{\circ} \mathrm{C}$ for $30 \mathrm{sec}$, annealing at $60^{\circ} \mathrm{C}$ for $30 \mathrm{sec}$ and extension at $70^{\circ} \mathrm{C}$ for $60 \mathrm{sec}$. A final extension was performed at $70^{\circ} \mathrm{C}$ for $5 \mathrm{~min}$.

Lab-on-a-chip genotyping was used as previously described to analyze the number of tandem repeats in each locus [18]. The amplification products were loaded into a 96-well or 384-well PCR plates that were prepared according to the manufacturer's recommendations (Caliper HT DNA 5 K Kit, Caliper Life Sciences, Hopkinton, USA). Each chip contained 5 active wells: 1 for the DNA marker and 4 for the gel-dye solution. A marker ladder of MW 100, 300, 500, 700, 1, 100, 1, 900, 2, 900 , and 4, 900 bp was used for referencing the molecular weight. The number of samples per chip preparation was 400, equivalent to four 96-well plates or one 384-well plate. After gel preparation, the sample plate was loaded into the plate carrier attached to the robot of the Caliper LabChip 90 (Caliper Life Sciences). During the separation of the fragments, the samples were analyzed sequentially, and electropherograms, virtual gel images and tabulated data were shown. The amplification product size estimates were obtained using the LabChip GX (Caliper Life Sciences) [18]. For each fragment size, the corresponding allele was assigned using the conversion table that was previously described [18]. The assigned number of each tandem repeat was imported into the BioNumerics software package (version 5.10, Applied Maths, Belgium). A clustering analysis was performed using the unweighted pair-group method using arithmetic averages (UPGMA). The UPGMA method assumes a constant rate of evolution, which is presumed for Brucella species because genetic recombination in Brucellae and horizontal gene transfer among Brucella species is low [32,33]. In all of the loci, the differences in the number of repeats were weighted equally because at one locus, multiple tandem repeats can be incorporated during one recombination event.

The publicly available MLVA database for Brucella (MLVA-NET for Brucella, http://mlva.u-psud.fr/brucella/) was used to identify or confirm the identity of all of the isolates used in this study. The comparison between the caliper data and MLVA bank showed some discrepancies for the allelic sequences that were obtained using different electrophoretic techniques. Due to the different nature of the gel matrix, these differences were resolved by sequencing $[18,30]$.

\section{Culture conditions and sample preparation for MALDI- TOF-MS analysis}

From a frozen stock, the bacteria were cultured on blood agar plates for at least $48 \mathrm{~h}$ at $35^{\circ} \mathrm{C}$ in the presence of $5 \%$ $\mathrm{CO}_{2}$. Before sample preparation, the isolates were regrown for $48 \mathrm{~h}$ at $35^{\circ} \mathrm{C}$ in the presence of $5 \% \mathrm{CO}_{2}$. Sample preparation was performed according to the company guidelines (Bruker Daltonics, Bremen, Germany). Briefly, 30 colonies were suspended in $300 \mu \mathrm{l}$ of water (MilliQ, Millipore, Billerica, MA, U.S.) and mixed carefully. Next, $900 \mu \mathrm{l}$ of absolute ethanol (Fisher Scientific, Loughborough, UK) was added and the suspension was mixed. Subsequently, the suspension was incubated for $90 \mathrm{~min}$ to inactivate all of the bacteria. After this inactivation step, the suspension samples were centrifuged for $10 \mathrm{~min}$ at $10,000 \mathrm{~g}$. The supernatant was removed. To remove the remaining ethanol residue, the spinning step was repeated, and the remaining supernatant was removed. Subsequently, $50 \mu \mathrm{l}$ of $70 \%$ formic acid was added to the pellet, and the pellet was mixed. Next, $50 \mu \mathrm{l}$ of pure acetonitrile (LC-MS grade, Fluka/Aldrich, Stenheim, Germany) was added, and the suspension was mixed carefully. The particulate matter that could not be dissolved was spun down by centrifugation for $2 \mathrm{~min}$ at 10 , $000 \mathrm{~g}$. Finally, four spots were created, using $0.5 \mu \mathrm{l}$ of the supernatant per spot, onto a MALDI-TOF target plate (MTP 384 target polished steel \#209519, Bruker Daltonics) and air dried. Subsequently, the spots were overlaid with $0.5 \mu \mathrm{l}$ of $\alpha$-cyano-4-hydroxycinnamic acid (HCCA, Bruker Daltonics) and a $10 \mathrm{mg} / \mathrm{ml}$ acetonitrile/ 
water solution (1:1) with $2.5 \%$ trifluoroacetic acid (TFA) (Fluka/Aldrich, Stenheim, Germany) and dried at room temperature.

\section{Mass spectra acquisition}

All of the mass spectra were automatically acquired on a Bruker Autoflex III smartbeam instrument (Bruker Daltonics $\mathrm{GmbH}$, Bremen, Germany) in linear mode using the following parameters: $40 \%$ laser intensity, positive polarity, $350 \mathrm{~ns}$ PIE delay, $20 \mathrm{kV}$ source voltage 1 , $18.7 \mathrm{kV}$ source voltage $2,8 \mathrm{kV}$ lens voltage, $1.522 \mathrm{kV}$ linear detector voltage, and $800 \mathrm{Da}$ detector gating. Composite mass spectra were generated from 10 different positions per spot using, in total, 2, 000 laser shots at each spot generated by a $200-\mathrm{Hz}$ smartbeam laser $(355 \mathrm{~nm})$. The mass spectra were recorded at a mass/ charge range between $800 \mathrm{Da}$ and $20 \mathrm{kDa}$. The instrument was externally calibrated with a bacterial test standard (BTS, Bruker). Furthermore, by including E. coli DH5 $\alpha$ during each extraction procedure, the complete procedure was validated. For the construction of the custom Brucella reference library, 24 MS spectra for each bacterium were generated (eight MS-spectra were generated per day on three different days).

\section{MALDI-TOF-MS data analyses}

The initial data analysis was performed with Bruker Daltonics MALDI Biotyper 2.0 software (Bruker). The raw spectra were automatically pre-processed in a 5-step approach: (1) mass adjustment, (2) smoothing, (3) baseline subtraction, (4) normalization, and (5) peak detection (Bruker). The MLVA genotyping results were used to set up a reference library for Brucella species. From each MLVA-cluster except cluster 8, one isolate was selected to generate a custom reference library for the identification of Brucella species (Table 1). For cluster 8, two isolates were selected because this cluster contained both $B$. suis and B. canis isolates. These isolates, 18 in total, were used to generate the Brucella reference library. From each selected isolate, a main spectra (MSP, a 'reference peak list' that is created using a fully automated process in Biotyper 2.0) was created using 24 MS spectra (from three independent measurements at eight different spots) according to company guidelines, using default settings (Bruker). A custom taxonomic tree was created based on the topology of the MLVA tree (Table 1). Subsequently, the MSPs were added to the corresponding taxon nodes. Next, from the remaining 152 isolates, four MS spectra were compared against the generated custom Brucella reference library, and the logarithmic score values were calculated. The logarithmic score value is determined by calculating the proportion of matching peaks and peak intensities between the test spectrum and the reference spectra of the database. The highest logarithmic score value is the closest match to a representative isolate in the reference library used. The logarithmic score values range from 0 to 3 . If the highest logarithmic score value is $<1.700$, the spectrum will be reported as 'not reliable identification', indicating that the spectrum could not be used to identify the strain with the reference library used. A logarithmic score value from 1.700 to 1.999 will be reported as 'probable genus identification', indicating that the genus identification is reliable. Next, a high logarithmic score value from 2.000 to 2.299 will be reported as 'secure genus identification, probable species identification', indicating that the genus identification is secure but that the species identification may be incorrect. A logarithmic score value of 2.300 to 3.000 will be reported as 'highly probable species identification', indicating that the isolate is identified at the species level with a high probability [27].

Apart from the Bruker Daltonics MALDI Biotyper 2.0 data analysis, for presentation purposes, the spectra were converted to the Matlab format. This conversion was performed in two steps: the spectra were first converted into the MZXML format, using the Bruker supplied executable CompassXport.exe, and subsequently to the Matlab binary format using the Matlab routine mzxmlread.m (Matlab 7.5). The spectra presented here were processed further using the Matlab Bioinformatics toolbox (Version 3.0) routines msresample.m for resampling, mslowess.m for smoothing, msbackadj.m for baseline subtraction and finally msnorm.m for normalization of the spectra.

\section{Results \\ MLVA}

The MLVA was used to ascertain the identity of all of the isolates used in this study by comparing their MLVA profiles against the publicly available MLVA database for Brucella (MLVA-NET for Brucella, http:// mlva.u-psud.fr/brucella/). All of the isolates except strain W99 were identified at the species level. Strain W99 matched as closely to a $B$. abortus as to a $B$. melitensis in the database, indicating the close relationship between the two species. This isolate is known in the literature as B. abortus W99, an A-epitope dominant strain used in a study in which the smooth lipopolysaccharides have been characterized [34]. This W99 strain differs at seven different loci from known $B$. melitensis and $B$. abortus isolates and thus is most likely an outlier.

The clustering of the MLVA results using the UPGMA clustering algorithm divided the 170 isolates into 14 clusters and 3 singletons with a genetic similarity of $>52.5 \%$ (Figures 1 and 2). The genetic relatedness of $>52.5 \%$ was somewhat arbitrarily selected based on the discriminatory power between species and/or biovars. In the dendrogram including the reference strains (Figures 1 and 2), all 
Table 1 Included strains and the taxonomic structure of the Brucella library generated using the Biotyper 2.0 program

\begin{tabular}{|c|c|c|c|c|c|}
\hline Genus & Group & Sub-group & MLVA cluster & Strain & Species \\
\hline \multirow[t]{18}{*}{ Brucella } & melitensis/abortus & melitensis & 1 & Ether & Brucella melitensis \\
\hline & & & 2 & $16 \mathrm{M}$ & Brucella melitensis \\
\hline & & & 3 & $63 / 9$ & Brucella melitensis \\
\hline & & abortus & 4 & $98 / 3033$ & Brucella abortus \\
\hline & & & 5 & W99 & Brucella abortus/melitensis \\
\hline & & & 6 & B19 & Brucella abortus \\
\hline & & & 7 & Tulya & Brucella abortus \\
\hline & non-melitensis/abortus & suis/canis/ovis & 8 & RM6/66 & Brucella canis \\
\hline & & & 8 & 686 & Brucella suis biovar 3 \\
\hline & & & 9 & S2 Chine & Brucella suis biovar 1 \\
\hline & & & 10 & Thomsen & Brucella suis biovar 2 \\
\hline & & & 11 & Réo 198 & Brucella ovis \\
\hline & & ceti/pinni/neo & 12 & 09-00388 & Brucella pinnipedialis \\
\hline & & & 13 & $17 g-1$ & Brucella pinnipedialis \\
\hline & & & 14 & $M 78 / 05 / 2$ & Brucella ceti \\
\hline & & & 15 & 513 & Brucella suis biovar 5 \\
\hline & & & 16 & M 644/93/1 & Brucella ceti \\
\hline & & & 17 & $5 K 33$ & Brucella neotomae \\
\hline
\end{tabular}

of the isolates clustered as expected from the literature and the species identification using MLVA (17). Although all of the reference strains were identified as the correct biovar, except for the identification of B. abortus biovar 5, which was identified as biovar 5 or 9 , identification to the biovar level using MLVA proved to be ambiguous because sometimes the profiles were found to be equally similar to multiple biovars. Thus, the biovar could not be assigned to 8 (29\%), 28 (30\%), and $2(11 \%)$ of the B. abortus, B. melitensis, and B. suis isolates, respectively. Cluster 10 only contained isolates of $B$. suis biovar 2. However, the other clusters contained multiple biovars. Based on genetic similarity, these clusters and the singletons could be divided into two genetically related groups. The first group, B. melitensis/abortus (BAM), consists of 6 clusters and 1 singleton (W99) isolate, which are all B. melitensis or B. abortus species. The second, non-BAM group is genetically more diverse and contains 8 clusters and 2 singletons comprising the other Brucella species (B. suis, B. canis, B. ovis, B. pinnipedialis, $B$. ceti, and B. neotomae). B. suis biovars 1,2 , and 3 and $B$. canis are genetically highly related, whereas $B$. suis biovar 5 is genetically distinct from other B. suis biovars. Epidemiologically related strains, from the same outbreak or isolated from the same patient, were grouped in the same clusters with a genetic relatedness of $70 \%$ or more (Figures 1 and 2).

\section{MALDI-TOF-MS}

The 608 MS spectra derived from 152, mostly clinical, isolates were compared against the reference library generated for Brucella species. Representative MS spectra from the 18 isolates selected for the Brucella reference library are shown (Figure 3 ). Minor visual differences (peaks and intensities) among the MS spectra are detectable. A total of 25 MS spectra had a logarithmic score value from 2.000 to 2.299 , indicating 'secure genus identification, probable species identification'. The highest logarithmic score values of the remaining 583 MS spectra were between 2.300 and 3.000, which indicate 'highly probable species identification'.

Because Brucella abortus W99, a singleton strain, is equally similar to $B$. abortus as to $B$. melitensis, we interpreted this strain as a potential B. melitensis strain. When identification at the species level is based on a 'majority rule' (i.e., identification is based on the species indicated by at least three out of four MS spectra), 149 $(98 \%)$ isolates were correctly identified at the species level. Further, when instead of the majority rule, the identification at the species level was based on the highest of the four logarithmic values, which was always $>2.299$, 151 (99.3\%) of the isolates were correctly identified at the species level, while only $1(0.7 \%)$ isolate was mistakenly identified as B. canis instead of B. suis.

The isolates 03-3081-2, 04-2987, and 02-00117, which were identified as B. suis biovar 3, 1 or 3 and 1 or 3 , respectively, based on their MLVA profile similarity, were all grouped into cluster 9, which only contained B. suis biovar 1 isolates. Therefore, these three isolates are most likely B. suis biovar 1 .

The MLVA data further demonstrated that the $B$. suis biovars 1 (MLVA cluster 9) and 2 (MLVA cluster $10)$ are genetically distinct clusters, whereas $B$. suis biovar 3 grouped together with $B$. canis isolates in a single 


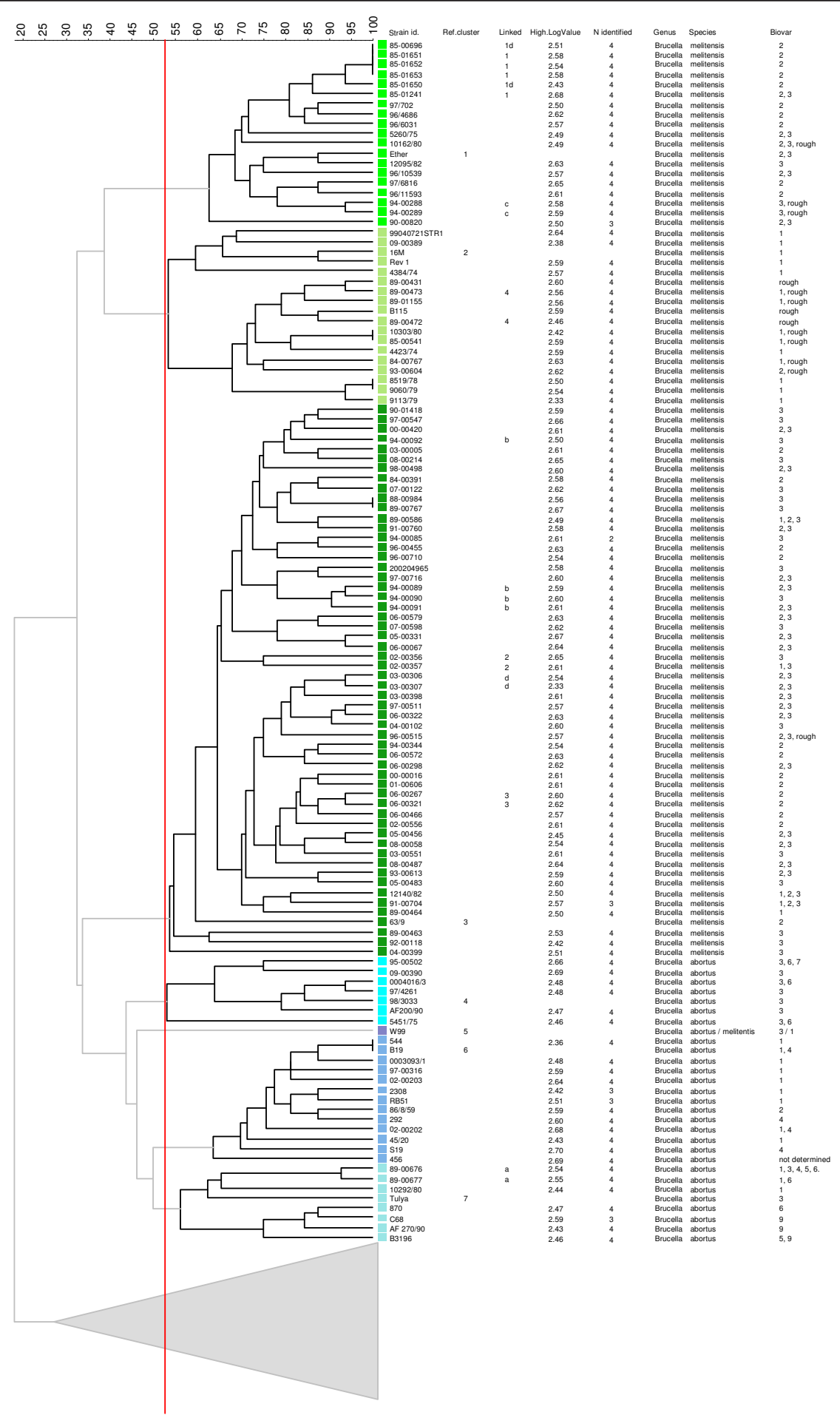

Figure 1 Partial dendrogram MLVA-16 clustering analysis of 170 Brucella isolates, with all 93 of the B. melitensis and 29 B. abortus isolates included in this study. The columns present the following data: original strain number [Strain id.], MLVA cluster number reference [Ref. cluster], epidemiologic relatedness (a-d indicate isolates from the same patient, 1-3 indicate isolates that are epidemiologically linked to each other)[Linked], highest logarithmic value of the four generated MS spectra [High LogValue], number of the 4 generated MS spectra corresponding with species identification using MLVA [N identified], genus [Genus], species [Species], and biovar [Biovar] identification based on the MLVA database. The similarity axis is presented in the top left corner. Each color reflects a different cluster with $>52.5 \%$ similarity. The group of 'melitensis-abortus' isolates clustered as follows: B. melitensis isolates grouped in Clusters 1, 2, and 3. B. abortus isolates grouped in Clusters 4, 6, and 7. Outlier B. abortus/melitensis W99 is a singleton (Cluster 5). 


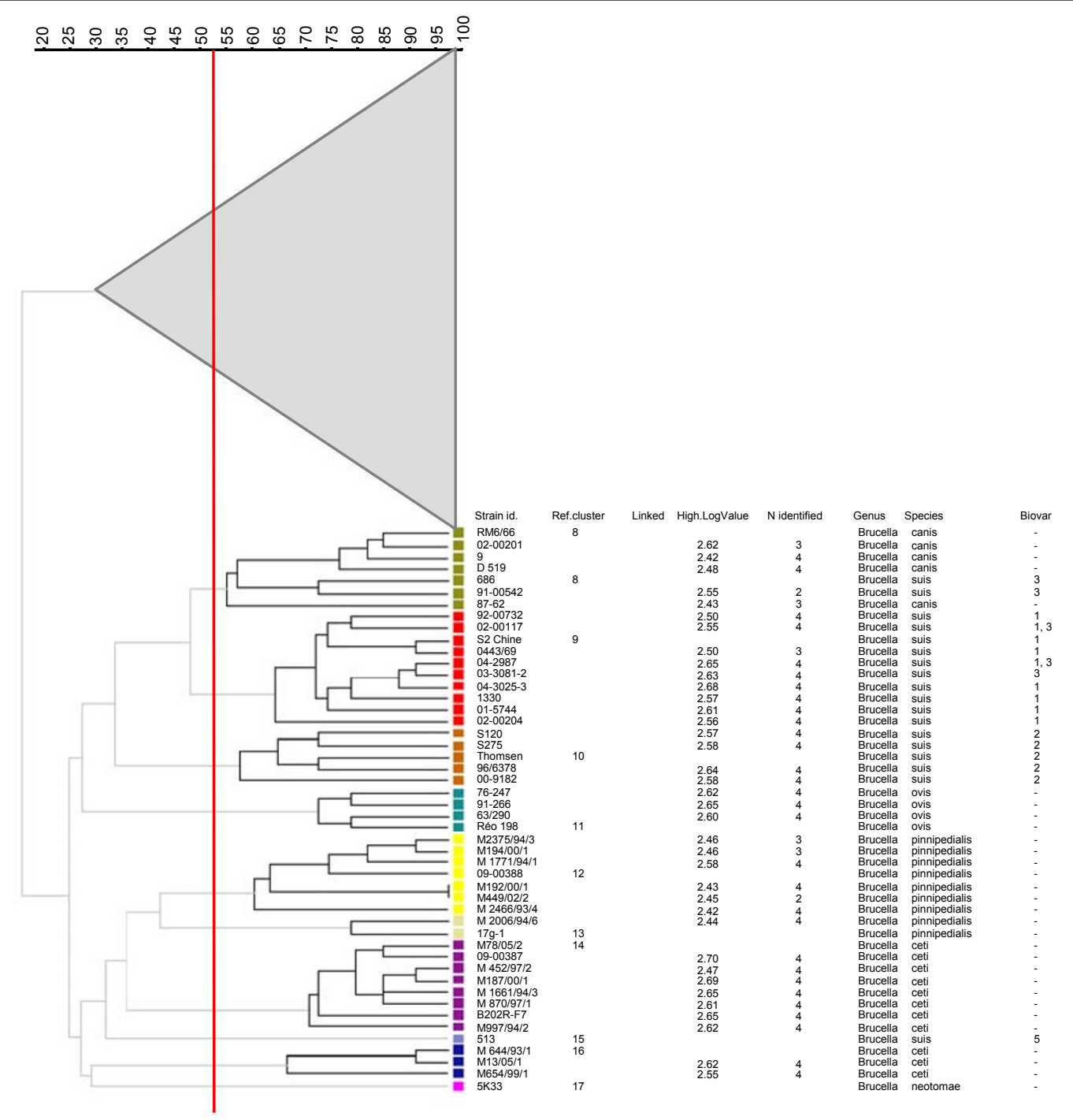

Figure 2 Partial dendrogram MLVA-16 clustering analysis of 170 Brucella isolates, including the $\mathbf{4 8}$ isolates from Brucella species that were not $\boldsymbol{B}$. melitensis or $\mathbf{B}$. abortus included in this study. The columns present data as described in Figure 1. The similarity axis is presented in the top left corner. Each color reflects a different cluster with $>52.5 \%$ similarity. The group of 'non-melitensis/abortus' isolates clustered as follows: Cluster 8 with B. suis biovar 3 and B. canis; Cluster 9 with B. suis biovar 1; Cluster 10 with B. suis biovar 2; and Cluster 11 with B. ovis isolates. The 'B. ceti/pinnipedialis/neotomae' subgroup clustered as follows: Clusters 12 and 13 with B. pinnipedialis isolates and Cluster 14 and 16 with B. ceti isolates. Furthermore, this subgroup also contained two clusters with only one isolate (singletons): Cluster 15 with a B. suis biovar 5 and Cluster 16 with a B. neotomae isolate.

genetic cluster (MLVA cluster 8). Next, we determined if the B. suis biovars could be identified to their biovar level using MALDI-TOF-MS. Of the 4 B. canis isolates and 14 B. suis isolates ( 9 were B. suis biovar 1 , assuming that the isolates 03-3081-2, 04-2987, and 02-00117 were biovar 1 as discussed previously, 4 were $B$. suis biovar 2 , and 1 was $B$. suis biovar 3 ), only the $B$. suis biovar 3 isolate was mistakenly identified as $B$. canis using either the 'majority' or 'highest score' rule. For these results, we have considered the library strain W99 to be B. melitensis. Removing W99 from the
Brucella reference library and comparing the 604 MS-spectra against this library only slightly influenced the classification results.

\section{Discussion}

An immediate response is required to mitigate the effects of a biological attack. The timely detection of a biological event is essential to respond. Then, exposure to the agent may be reduced by the application of protective measures, the most important of which is airway protection. B. melitensis, B. suis, and possibly B. abortus are 


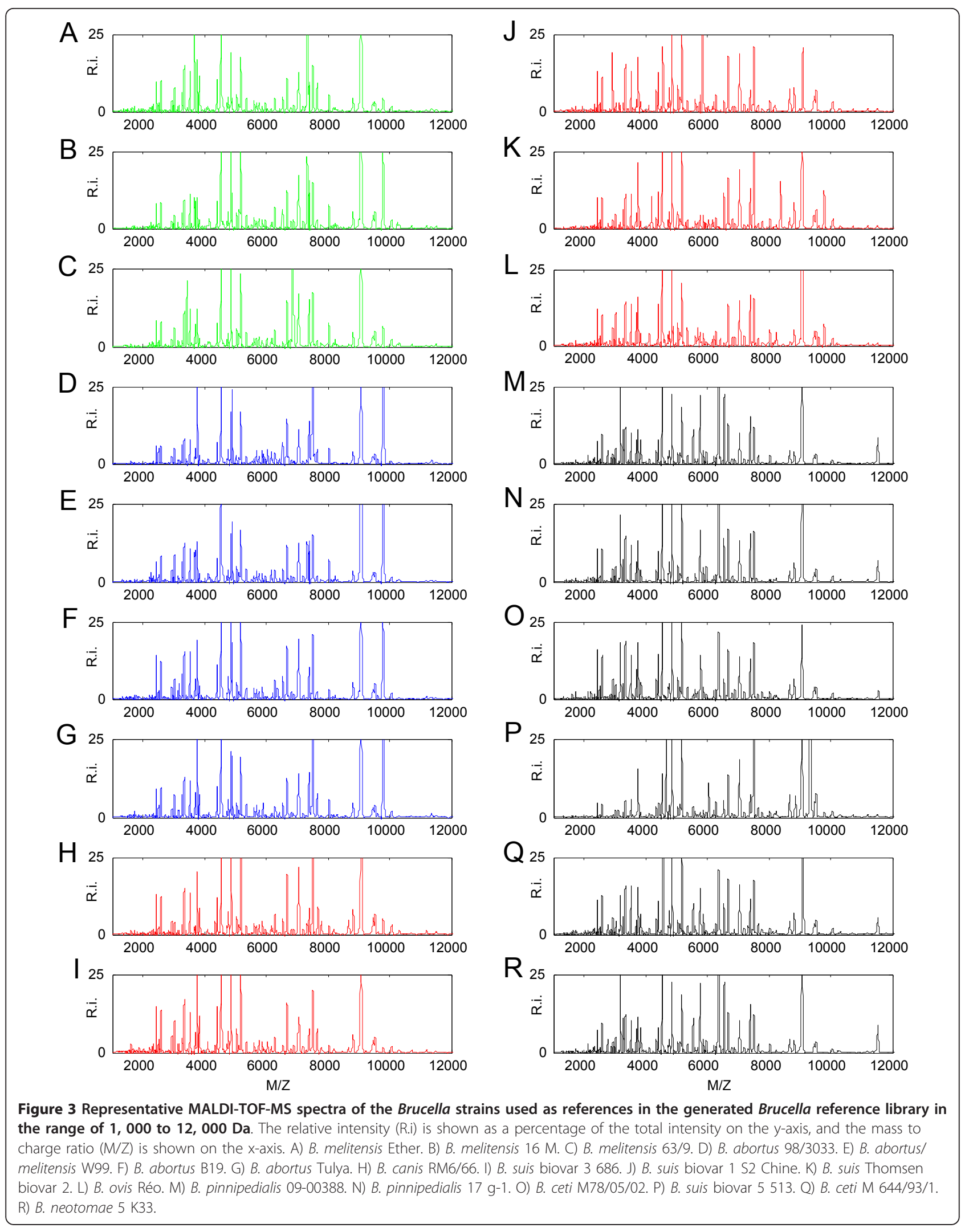


considered to be potential warfare agents. To date, the detection and identification of Brucella species is laborious and time consuming. However, MALDI-TOF-MS may provide a new and rapid method that enables the quick identification of microorganisms. Brucella species are very difficult to identify. Not only are the species genetically highly related but also the taxonomy of Brucella species is open to debate because discrepancies in the nomenclature used were observed in the past [33]. First, $B$. suis is paraphyletic, from a genetic point of view because it contains not only B. suis but also B. canis [32]. Further, whole-genome sequencing demonstrated that $B$. canis is genetically highly similar to $B$. suis biovars 3 and 4 [32]. Likely, B. canis has arisen from its ancestor $B$. suis. In contrast, $B$. suis biovar 5 is genetically much more related to $B$. pinnipedialis and $B$. ceti than to the other B. suis biovars [19,32]. Second, Maquart and coworkers showed that $B$. ceti is divided into two separate clusters, one cluster of which was genetically more related to $B$. pinnipedialis than to the other cluster of $B$. ceti [20]. Third, B. melitensis from the western Mediterranean is genetically closer to $B$. abortus than to $B$. melitensis of eastern Mediterranean or American origin [20]. Clearly, the taxonomy of Brucella species is based on pathogenesis, host specificity, and geographic source rather than on genetic relationships. These issues complicate the development of new identification methods but also complicate the interpretation of the identification results, which is illustrated by the fact that no specific biological markers for $B$. suis have been identified $[14,33]$. A new classification, based on genetics, of the taxa within the genus Brucella is needed, rather than assigning the names of the conventional species and biotypes to the taxa created using molecular methods. Only species and biotypes that are both genotypically and phenotypically coherent can be maintained. Because this reclassification is beyond the scope of this article, the identification of the Brucellae used in this study was based on the MLVA database.

The previously developed 16-MLVA method has been shown to have a high discriminatory power and is able to correctly identify all of the known species of the Brucella genus $[13,18-20]$. Therefore, identification at the species level of isolates based on comparisons with the MLVA database should be considered reliable. However, identification at the biovar level using MLVA analysis proved to be ambiguous, especially for B. melitensis and B. abortus, as described previously $(1,14)$. Although we found some discrepancies in the MLVA profiles of the reference strains between the publically available database and our results, these differences are likely due to difficulties in the interpretation of the MLVA profiles because of the small and contiguous sizes of some alleles (Bruce 08, 21, 16 and 19).
In this study, we demonstrated that MALDI-TOF-MS enables the identification of Brucella isolates at the species level. Predominantly, isolates of B. melitensis and B. abortus, the main cause of human brucellosis in The Netherlands, were tested, and all of the isolates were identified correctly. Although the number of B. suis biovar 1 and 2 isolates in this study was limited, the isolates present were correctly identified at their biovar level as well. The interpretation of the one isolate of B. suis biovar 3 as $B$. canis is likely due to the high similarity of $B$. suis biovars 3 and 4 to $B$. canis [32]. A previous study by Ferreira et al. could not discriminate at the species level [25]. The constructed reference library by Ferreira et al. did not represent the complete diversity between Brucella species, which could possibly explain the reduced discriminatory power to the species level. Furthermore, we noticed that strain NCTC 10098 was a B. melitensis according the NCTC and not a B. suis as it has been used by Ferreira et al. [25]. In addition, in the library of Ferreira et al., no B. abortus isolates of cluster 4 (Figure 1) were included.

This study presents an additional observation that further highlights the controversy of combining molecular data with the conventional taxonomy of the genus Brucella. As mentioned earlier, the results described are based on the assumption that the B. abortus strain W99 is phenotypically more strongly related to $B$. melitensis than to $B$. abortus. This assumption was supported by the results because the MS spectra of the 80 isolates that were identified to be $B$. melitensis using MLVA closely resembled the MS spectrum of W99, whereas none of the MS spectra derived from $B$. abortus isolates had a similar resemblance. Thus, phenotypically, strain W99 is more closely related to $B$. melitensis than to $B$. abortus. It is possible that strain W99 is related to the common ancestor of the BAM group.

MALDI-TOF-MS identification is based on profiles derived from the proteome. To counter the inherent minor variations found between measurements of the MS spectra, the MS profiles in the reference library constructed here consist of the mean of 24 MS spectra. The fact that the identification of genetically highly related species appeared to be feasible demonstrates that even minor genetic differences are translated to specific proteomic differences.

\section{Conclusions}

Discrepancies between classical taxonomy and the genetic relatedness of species and biovars complicate the development of detection and identification assays. Despite these difficulties, the accurate identification of Brucella species was achieved with MALDI-TOF-MS by constructing a Brucella reference library based on genetic relationships according to MLVA data. We conclude that MALDI-TOF-MS can be developed into a 
fast and reliable identification method for genetically highly related species when potential taxonomic and genetic inconsistencies are considered during the generation of the reference library.

\section{Additional material}

Additional file 1: Table S1. Strains used during the study with additional information.

\section{Acknowledgements}

We wish to thank K. Walravens for providing strains and D. van der Kleij for her comments and critical reading of the manuscript.

This work was financially supported by the Dutch Ministry of Defense, grant number V1036. This work was part of the European Defence Agency (EDA) project B0060 involving biodefence institutions from Italy and The Netherlands.

\section{Author details}

'Department of Earth, Environmental, and Life Sciences, TNO, Lange Kleiweg 137, Rijswijk, P.O Box 45, The Netherlands. ${ }^{2}$ Department of Bacteriology, Diagnostic Laboratory for Infectious Diseases and Perinatal Screening, National Institute of Public Health and the Environment (RIVM), Antonie van Leeuwenhoeklaan 9, Bilthoven 3721, MA, The Netherlands. ${ }^{3}$ Histology and Molecular Biology Section, Army Medical and Veterinary Research Center, Via Santo Stefano Rotondo 4, 00184 Rome, Italy. ${ }^{4}$ Military Health Care Expertise Co-ordination Centre, Ministry of Defence, Noodweg 37, Hilversum 1213, PW, The Netherlands.

\section{Authors' contributions}

FL participated in the design of the study and coordinated the MLVA work; FR participated in the design of the study and critically revised the manuscript; RDS executed the MLVA experiments, analyzed the data and drafted the manuscript; RP participated in the analysis of the MALDI-TOF-MS data; AdJ executed the MALDI-TOF-MS experiments and participated in the MALDI-TOF-MS data analysis; JK participated in the design of the study; AvdL executed the MALDI-TOF-MS experiments; IW executed the MALDITOF-MS experiments; SF executed the MLVA experiments; HJJ participated in the design of the study and critically revised the manuscript; JVdP participated in the design of the study and critically revised the manuscript; and AP participated in the design of the study, performed data analysis on the MLVA and MALDI-TOF-MS data, coordinated the MALDI-TOF-MS experiments, and drafted the manuscript. All authors read and approved the final manuscript.

Received: 4 August 2011 Accepted: 23 December 2011

Published: 23 December 2011

\section{References}

1. Brenner DJ, Krieg NR, Staley JT, Corbel MJ, Banai M: Bergey's Manual of Systemic Bacteriology. In Volume 2 part C.. 2 edition. Edited by: Corbel MJ, Banai M. New York: Springer Science; 2005:370-386.

2. Franz DR, Jahrling PB, Friedlander AM, McClain DJ, Hoover DL, Bryne WR, Pavlin JA, Christopher GW, Eitzen EM Jr: Clinical recognition and management of patients exposed to biological warfare agents. JAMA 1997, 278:399-411.

3. Yagupsky P, Baron EJ: Laboratory exposures to brucellae and implications for bioterrorism. Emerg Infect Dis 2005, 11:1180-1185.

4. Yingst SL, Huzella LM, Chuvala L, Wolcott M: A rhesus macaque (Macaca mulatta) model of aerosol-exposure brucellosis (Brucella suis): pathology and diagnostic implications. J Med Microbiol 2010, 59:724-730.

5. Cloeckaert A, Verger JM, Grayon M, Paquet JY, Garin-Bastuji B, Foster G, Godfroid J: Classification of Brucella spp. isolated from marine mammals by DNA polymorphism at the omp locus. Microbes Infect 2001, 3:729-738.

6. Foster G, Osterman BS, Godfroid J, Jacques I, Cloeckaert A: Brucella cet sp. nov. and Brucella pinnipedialis sp. nov. for Brucella strains with cetaceans and seals as their preferred hosts. Int I Syst Evol Microbiol 2007, 57:2688-2693.

7. Jahans KL, Foster G, Broughton ES: The characterisation of Brucella strains isolated from marine mammals. Vet Microbiol 1997, 57:373-382.

8. Scholz HC, Hubalek Z, Nesvadbova J, Tomaso H, Vergnaud G, Le Fleche P, Whatmore AM, Al Dahouk S, Kruger M, Lodri C, et al: Isolation of Brucella microti from soil. Emerg Infect Dis 2008, 14:1316-1317.

9. Scholz HC, Hubalek Z, Sedlacek I, Vergnaud G, Tomaso H, Al Dahouk S, Melzer F, Kampfer P, Neubauer H, Cloeckaert A, et al: Brucella microti sp. nov., isolated from the common vole Microtus arvalis. Int I Syst Evol Microbiol 2008, 58:375-382.

10. Scholz HC, Hofer E, Vergnaud G, Le Fleche P, Whatmore AM, Al Dahouk S, Pfeffer M, Kruger M, Cloeckaert A, Tomaso H: Isolation of Brucella microti from mandibular lymph nodes of red foxes, Vulpes vulpes, in lower Austria. Vector Borne Zoonotic Dis 2009, 9:153-156.

11. Scholz HC, Nockler K, Gollner C, Bahn P, Vergnaud G, Tomaso H, Al Dahouk S, Kampfer P, Cloeckaert A, Maquart M, et al: Brucella inopinata sp. nov., isolated from a breast implant infection. Int I Syst Evol Microbiol 2010, 60:801-808.

12. Tiller RV, Gee JE, Lonsway DR, Gribble S, Bell SC, Jennison AV, Bates J, Coulter C, Hoffmaster AR, De BK: Identification of an unusual Brucella strain (BO2) from a lung biopsy in a 52 year-old patient with chronic destructive pneumonia. BMC Microbiol 2010, 10:23.

13. Whatmore AM: Current understanding of the genetic diversity of Brucella, an expanding genus of zoonotic pathogens. Infect Genet Evol 2009, 9:1168-1184.

14. Moreno E, Cloeckaert A, Moriyon I: Brucella evolution and taxonomy. Vet Microbiol 2002, 90:209-227.

15. Verger $\mathrm{JM}$, Grayon $\mathrm{M}$, Cloeckaert A, Lefevre $\mathrm{M}$, Ageron E, Grimont F: Classification of Brucella strains isolated from marine mammals using DNA-DNA hybridization and ribotyping. Res Microbiol 2000, 151:797-799.

16. Lopez-Goni I, Garcia-Yoldi D, Marin CM, de Miguel MJ, Munoz PM, Blasco JM, Jacques I, Grayon M, Cloeckaert A, Ferreira AC, et al: Evaluation of a multiplex PCR assay (Bruce-ladder) for molecular typing of all Brucella species, including the vaccine strains. J Clin Microbiol 2008, 46:3484-3487.

17. Top J, Schouls LM, Bonten MJ, Willems RJ: Multiple-locus variable-number tandem repeat analysis, a novel typing scheme to study the genetic relatedness and epidemiology of Enterococcus faecium isolates. J Clin Microbiol 2004, 42:4503-4511.

18. De Santis R, Ciammaruconi A, Faggioni G, Fillo S, Gentile B, Di Giannatale E, Ancora M, Lista F: High throughput MLVA-16 typing for Brucella based on the microfluidics technology. BMC Microbiol 2011, 11:60.

19. Le Fleche P, Jacques I, Grayon M, Al Dahouk S, Bouchon P, Denoeud F, Nockler K, Neubauer H, Guilloteau LA, Vergnaud G: Evaluation and selection of tandem repeat loci for a Brucella MLVA typing assay. BMC Microbiol 2006, 6:9

20. Maquart M, Le Fleche P, Foster G, Tryland M, Ramisse F, Djonne B, Al Dahouk S, Jacques I, Neubauer H, Walravens K, et al: MLVA-16 typing of 295 marine mammal Brucella isolates from different animal and geographic origins identifies 7 major groups within Brucella ceti and Brucella pinnipedialis. BMC Microbiol 2009, 9:145.

21. Seng P, Drancourt M, Gouriet F, La Scola B, Fournier PE, Rolain JM, Raoult D: Ongoing revolution in bacteriology: routine identification of bacteria by matrix-assisted laser desorption ionization time-of-flight mass spectrometry. Clin Infect Dis 2009, 49:543-551.

22. Cherkaoui A, Hibbs J, Emonet S, Tangomo M, Girard M, Francois $\mathrm{P}$, Schrenzel J: Comparison of two matrix-assisted laser desorption ionization-time of flight mass spectrometry methods with conventional phenotypic identification for routine identification of bacteria to the species level. J Clin Microbiol 2010, 48:1169-1175.

23. Mellmann A, Bimet F, Bizet C, Borovskaya AD, Drake RR, Eigner U, Fahr AM, He Y, llina EN, Kostrzewa M, et al: High interlaboratory reproducibility of matrix-assisted laser desorption ionization-time of flight mass spectrometry-based species identification of nonfermenting bacteria. J Clin Microbiol 2009, 47:3732-3734.

24. van Veen SQ, Claas EC, Kuijper EJ: High-throughput identification of bacteria and yeast by matrix-assisted laser desorption ionization-time of flight mass spectrometry in conventional medical microbiology laboratories. J Clin Microbiol 2010, 48:900-907.

25. Ferreira L, Vega CS, Sanchez-Juanes F, Gonzalez-Cabrero S, Menegotto F, Orduna-Domingo A, Gonzalez-Buitrago JM, Munoz-Bellido JL: Identification 
of Brucella by MALDI-TOF mass spectrometry. Fast and reliable identification from agar plates and blood cultures. PLoS One 2010, 5: e14235.

26. Lasch P, Beyer W, Nattermann H, Stammler M, Siegbrecht E, Grunow R, Naumann D: Identification of Bacillus anthracis by using matrix-assisted laser desorption ionization-time of flight mass spectrometry and artificial neural networks. Appl Environ Microbiol 2009, 75:7229-7242.

27. Seibold E, Maier T, Kostrzewa M, Zeman E, Splettstoesser W: Identification of Francisella tularensis by whole-cell matrix-assisted laser desorption ionization-time of flight mass spectrometry: fast, reliable, robust, and cost-effective differentiation on species and subspecies levels. J Clin Microbiol 2010, 48:1061-1069.

28. Vanlaere E, Sergeant K, Dawyndt P, Kallow W, Erhard M, Sutton H, Dare D, Devreese B, Samyn B, Vandamme P: Matrix-assisted laser desorption ionisation-time-of of-flight mass spectrometry of intact cells allows rapid identification of Burkholderia cepacia complex. J Microbiol Methods 2008, 75:279-286.

29. Al Dahouk S, Fleche PL, Nockler K, Jacques I, Grayon M, Scholz HC, Tomaso H, Vergnaud G, Neubauer H: Evaluation of Brucella MLVA typing for human brucellosis. J Microbiol Methods 2007, 69:137-145.

30. De Santis R, Ciammaruconi A, Faggioni G, D'Amelio R, Marianelli C, Lista F: Lab on a chip genotyping for Brucella spp. based on 15-loci multi locus VNTR analysis. BMC Microbiol 2009, 9:66.

31. Kattar MM, Jaafar RF, Araj GF, Le Fleche P, Matar GM, Abi RR, Khalife S, Vergnaud $\mathrm{G}$ : Evaluation of a multilocus variable-number tandem-repeat analysis scheme for typing human Brucella isolates in a region of brucellosis endemicity. J Clin Microbiol 2008, 46:3935-3940.

32. Foster JT, Beckstrom-Sternberg SM, Pearson T, Beckstrom-Sternberg JS, Chain PS, Roberto FF, Hnath J, Brettin T, Keim P: Whole-genome-based phylogeny and divergence of the genus Brucella. J Bacteriol 2009, 191:2864-2870.

33. Whatmore AM, Perrett LL, MacMillan AP: Characterisation of the genetic diversity of Brucella by multilocus sequencing. BMC Microbiol 2007, 7:34.

34. Weynants V, Gilson D, Cloeckaert A, Tibor A, Denoel PA, Godfroid F, Limet JN, Letesson JJ: Characterization of smooth lipopolysaccharides and $\mathrm{O}$ polysaccharides of Brucell species by competition binding assays with monoclonal antibodies. Infect Immun 1997, 65:1939-1943.

doi:10.1186/1471-2180-11-267

Cite this article as: Lista et al:: Reliable identification at the species level of Brucella isolates with MALDI-TOF-MS. BMC Microbiology 2011 11:267.

\section{Submit your next manuscript to BioMed Central and take full advantage of:}

- Convenient online submission

- Thorough peer review

- No space constraints or color figure charges

- Immediate publication on acceptance

- Inclusion in PubMed, CAS, Scopus and Google Scholar

- Research which is freely available for redistribution 